\title{
Direct quantification of transendothelial electrical resistance in organs-on-chips
}

Marinke W. van der Helm, ${ }^{a, *}$ Mathieu Odijk, ${ }^{a}$ Jean-Philippe Frimat, ${ }^{b}$ Andries $D$. van der Meer, ${ }^{c} \mathrm{Jan}$

C.T. Eijkel, ${ }^{a}$ Albert van den Berg ${ }^{a} \&$ Loes I. Segerink a

a BIOS Lab on a Chip group, MIRA Institute for Biomedical Technology and Technical Medicine \& MESA+ Institute for Nanotechnology, University of Twente, P.O. box 217, 7500 AE Enschede, The Netherlands.

${ }^{b}$ Microsystems, Eindhoven University of Technology, P.O. box 513, 5600 MB Eindhoven, the Netherlands.

${ }^{c}$ Applied Stem Cell Technologies, MIRA Institute for Biomedical Technology and Technical Medicine, University of Twente, P.O. box 217, 7500 AE Enschede, The Netherlands.

*Corresponding author: m.w.vanderhelm@utwente.nl (M.W. van der Helm).

\begin{abstract}
Measuring transendothelial or transepithelial electrical resistance (TEER) is a widely used method to monitor cellular barrier tightness in organs-on-chips. Unfortunately, integrated electrodes close to the cellular barrier hamper visual inspection of the cells or require specialized cleanroom processes to fabricate see-through electrodes. Out-of-view electrodes inserted into the chip's outlets are influenced by the fluid-filled microchannels with relatively high resistance. In this case, small changes in temperature or medium composition strongly affect the apparent TEER. To solve this, we propose a simple and universally applicable method to directly determine the TEER in microfluidic organs-on-chips without the need for integrated electrodes close to the cellular barrier. Using four electrodes inserted into two channels - two on each side of the porous membrane - and six different measurement configurations we can directly derive the isolated TEER independent of channel properties. We show that this method removes large variation of non-biological origin in chips filled with culture medium. Furthermore, we demonstrate the use of our method by quantifying the TEER of a monolayer of human hCMEC/D3 cerebral endothelial cells, mimicking the blood-brain barrier inside our microfluidic organ-on-chip device. We found stable TEER values of $22 \Omega \mathrm{cm}^{2} \pm 1.3 \Omega \mathrm{cm}^{2}$ (average \pm standard error of the mean of 4 chips), comparable to other TEER values reported for hCMEC/D3 cells in well-established Transwell systems. In conclusion, we demonstrate a simple and robust way to directly determine TEER that is applicable to any organ-on-chip device with two channels separated by a membrane. This enables stable and easily applicable TEER measurements without the need for specialized cleanroom processes and with visibility on the measured cell layer.
\end{abstract}

\section{Keywords}

Transendothelial electrical resistance; Transepithelial electrical resistance; Organs-on-chips; Blood-brain barrier on chip; Microfluidics

\section{Introduction}

Transendothelial or transepithelial electrical resistance (TEER) is a powerful, non-invasive measure of the tightness of a cellular barrier in vitro. It is widely used in both conventional Transwell culture systems and in recently developed organs-on-chips to monitor barrier formation and function (Abbott et al. 2014; Cardoso et al. 2010; Odijk et al. 2015; Srinivasan et al. 2015; Thuenauer et al. 2014). Organs-on-chips are microfluidic devices in which human tissues can be cultured in a controlled environment that is 
engineered to better mimic the physiologically relevant in vivo microenvironment of that specific tissue than standard in vitro culture systems (Bhatia and Ingber 2014; Moraes et al. 2012; van der Meer and van den Berg 2012). Generally, these devices comprise two microfluidic channels that are separated by a porous membrane on which the barrier-forming tissue is cultured. To measure the TEER of a cellular barrier inside such an organ-on-chip device, there have been reports of devices with integrated electrodes on either side of the porous membrane close to the cellular barrier (Booth and Kim 2012; Douville et al. 2010; Griep et al. 2013; Walter et al. 2016). However, these electrodes often hamper visual inspection of the cells and need to be fabricated and carefully aligned in a cleanroom environment (Booth and Kim 2012; Douville et al. 2010; Griep et al. 2013). To solve this, optically transparent electrodes have been reported (Walter et al. 2016), but these require precise sputtering of a gold layer with only $25 \mathrm{~nm}$ thickness, for which specialized cleanroom equipment is needed.

Alternatively, there are reports of electrodes being inserted in the outlets of organs-on-chips, analogous to the common method for the conventional in vitro Transwell system. This method relies on standard manufacturing techniques, is easy to perform experimentally and still allows optical access to the cells inside the chip (Brown et al. 2015; Deosarkar et al. 2015; Ferrell et al. 2010; Huh et al. 2010; Kim et al. 2012). However, the reliability and stability of TEER measurements that are performed with this method are lower, due to the small channel geometries of organ-on-chip systems. To illustrate this, the electrical resistance of an electrolyte, such as cell culture medium, in a microfluidic channel can be approximated with Eq. 1 (assuming uniform current density).

$$
R_{c h}=\rho \frac{l_{c h}}{A_{c h}}[\Omega]
$$

In this formula, $R_{c h}$ is the electrical resistance of the electrolyte inside a microfluidic channel $(\Omega), \rho$ is the specific electrical resistance of the electrolyte $(\Omega \mathrm{m}), l_{c h}$ is the length of the channel $(\mathrm{m})$ and $A_{c h}$ is the cross-sectional area of the channel $\left(\mathrm{m}^{2}\right)$. Using this formula it can be easily seen that the small cross-sectional area $A_{c h}$ and the relatively long channel length $l_{c h}$ result in a high resistance $R_{c h}$ for microfluidic channels. Variations in the measured resistance can easily arise from differences in electrode position when the electrodes are reinserted, resulting in a different channel length $l_{c h}$. In addition, area $A_{c h}$ can change in a part of the channel when air bubbles or other non-conducting inhomogeneities are present. Furthermore, the specific electrical resistance $\rho$ is a material property of the electrolyte, depending on electrolyte composition and temperature. Therefore, measuring at e.g. different temperatures or at a higher ion concentration as a result of evaporation results in variations in $\rho$ and thus in the measured resistance. In conclusion, all these variations add up to the measured TEER and can therefore wrongly suggest that the tightness of the cellular barrier changes, while changes of non-biological origin are the actual cause. Therefore, $l_{c h}, A_{c h}$ and $\rho$ need to be tightly controlled to achieve reliable resistance measurements using the conventional methods. Lack of control and standardization can be a cause of the large variation in TEER reported for the same cell type in the standard Transwell system (Odijk et al. 2015). In addition, TEER measurements were conducted in only four out of the ten organs-on-chips summarized in van der Helm et al. (2016), illustrating the challenge of incorporating TEER measurements into organs-on-chips.

To overcome this, we present a simple and universally applicable method to directly determine the TEER in microfluidic organs-onchips without the need for integrated electrodes close to the cellular barrier. Using four electrodes inserted into the two channels two on each side of the membrane - and six measurements we can directly determine the TEER. To illustrate the applicability of this method, we mimic the blood-brain barrier (BBB) inside our microfluidic organ-on-chip device using a human microvascular cerebral endothelial cell line (hCMEC/D3). The BBB is primarily formed by a tight monolayer of endothelial cells, which are linked to 
each other by tight junction proteins (e.g. zonula occludens-1 proteins; ZO-1). The BBB regulates transport of molecules and cells between blood and brain, providing an optimal environment for neuronal function (Abbott et al. 2010), and BBB dysfunction is involved in various neurological disorders (van der Meer et al. 2014). Our presented method is applicable to any organ-on-chip device with two channels that are separated by a porous membrane, making standardized TEER measurements more easily available for organ-on-chip applications.

\section{Materials and methods}

\subsection{Chip fabrication}

Polydimethylsiloxane (PDMS) base agent and curing agent were mixed in a 10:1 weight ratio (Sylgard 184 Silicone elastomer kit, Dow Corning, Midland, MI, USA). After degassing, this mixture was poured onto an SU-8 patterned silicon wafer and cured for 4 hours at $60^{\circ} \mathrm{C}$. The PDMS with channel imprints was cut into top and bottom parts, and four inlets (1.2 mm diameter) were punched into the top parts. Dust was removed using Scotch tape (3M). Leakage-free bonding of the two PDMS parts with a membrane in between was achieved by using a PDMS/ toluene mortar at 5:3 weight ratio (toluene from Merck, Germany) (Chueh et al. 2007; Griep et al. 2013). This mixture was spin-coated onto a glass coverslip (1500 rpm for $60 \mathrm{~s}$, ramped at $1000 \mathrm{rpm} \mathrm{s}{ }^{-1}$; Spin 150, Polos, the Netherlands) and a thin layer was transferred to both PDMS halves using an ink roller. A porous membrane of $4 \mathrm{~mm}^{2}$, cut from polycarbonate membranes with $0.4 \mu \mathrm{m}$ pores (Transwell culture inserts, Corning Incorporated) was placed in the centre of the bottom halve. The top part was aligned and gently placed on the bottom part without applying pressure, after which the chips were baked for 3 hours at $60^{\circ} \mathrm{C}$. Lastly, four platinum wires (200 $\mu$ m diameter; Alfa Aesar, Karlsruhe, Germany) were inserted into the electrode channels of the chip and fixed using Norland Optical Adhesive 81 (NOA81, Cranbury, NJ, USA), by applying a drop of NOA at the channel's entrance and curing it with UV (365 nm for $5 \mathrm{~s}$ at $350 \mathrm{~mW} \mathrm{~cm}^{-2}$ ) as soon as the channel was filled by capillary forces. The electrodes were inserted about $1 \mathrm{~mm}$ into the microchannel to ensure the exposure of a sufficiently large surface area to culture medium, which resulted in a large enough electrical double layer capacitance to not influence the TEER readout (see Suppl. Fig. 1). The fully assembled chip was placed on a plastic dish and fixed with a 2component epoxy adhesive (Loctite M-31 CL Hysol, Henkel) to prevent the electrodes from being pulled out of the chip during measurements. To cure the NOA and Hysol the chips were baked for 2 hours at $60^{\circ} \mathrm{C}$. The chip design and assembled chip are shown in Fig. 1. 

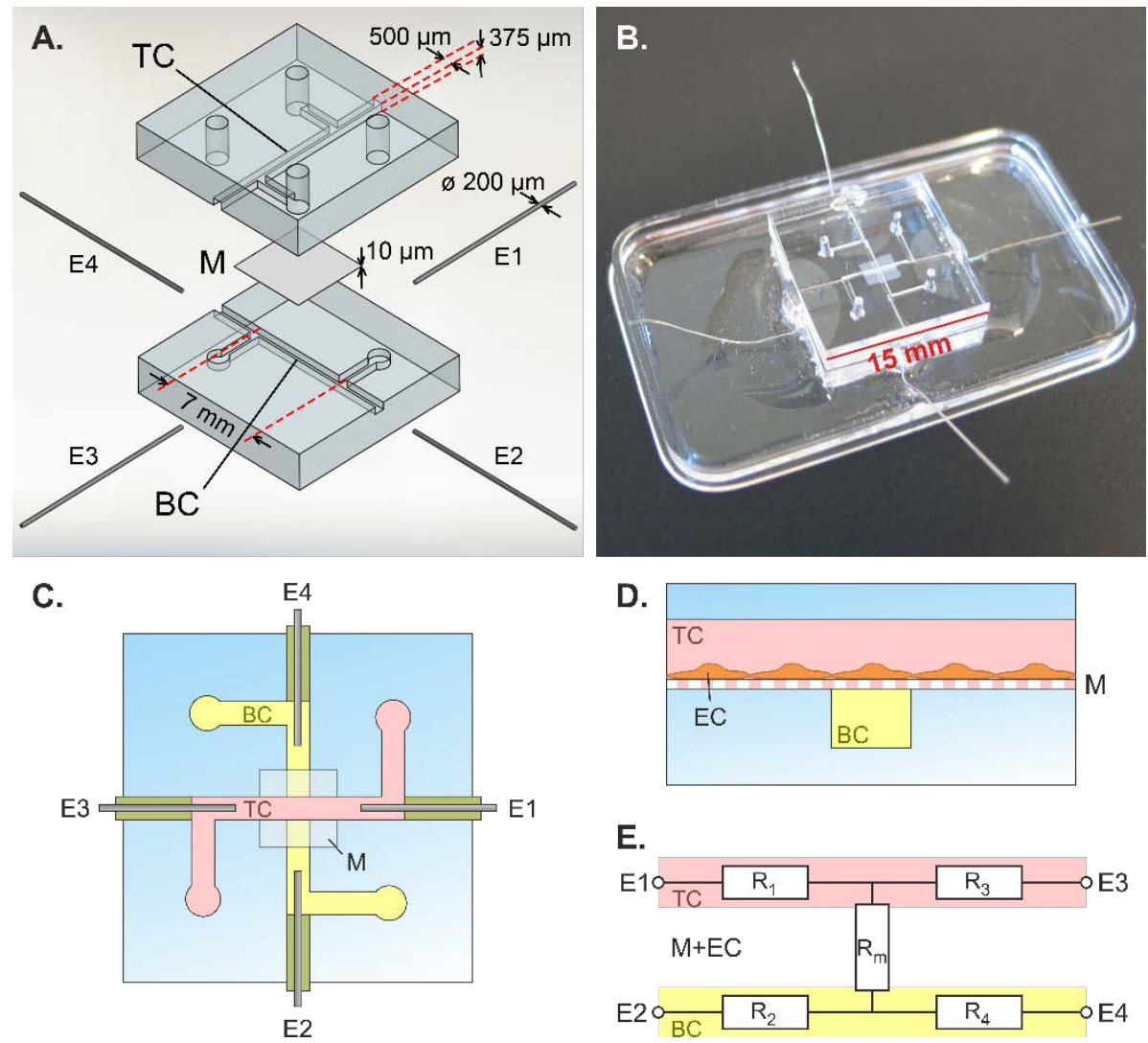

D.

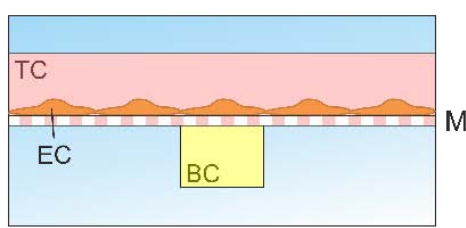

E.

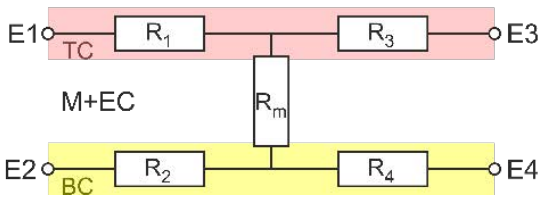

Fig. 1 Chip design. A. Exploded view of the PDMS chip with the top channel (TC), membrane (M), bottom channel (BC) and platinum wire electrodes (E1, E2, E3, E4). The culture area A on the membrane suspended between both channels is $0.25 \mathrm{~mm}^{2}$. B. Picture of the assembled chip. C. Schematic top view of the chip. D. Schematic cross section of the chip at the center, showing endothelial cells (EC) on membrane $M$ in the TC. E. Simplified equivalent circuit of the chip, showing the electrodes E1-E4, resistors representing the top channel $\left(R_{1}\right.$ and $\left.R_{3}\right)$, resistors representing the bottom channel $\left(R_{2}\right.$ and $\left.R_{4}\right)$ and resistor $R_{m}$ representing the membrane and EC barrier.

\subsection{Cell culture and staining}

The cells used in the BBB chip are immortalized human cerebral microvascular endothelial cells (hCMEC/D3 cell line, kindly provided by Dr. P.-O. Couraud, INSERM, Paris, France) (Weksler et al. 2005). hCMEC/D3 cells were cultured with endothelial growth medium (EGM-2: EBM-2 with EGM-2 SingleQuots, Lonza) in T75 culture flasks, coated with $20 \mu \mathrm{g} \mathrm{mL}{ }^{-1}$ human plasma fibronectin (Gibco) in phosphate buffered saline (PBS; Sigma) for $>30$ minutes at $37^{\circ} \mathrm{C}$. The cells were incubated at $37^{\circ} \mathrm{C}$ in humidified air with $5 \% \mathrm{CO}_{2}$. Medium was refreshed every 2-3 days and confluent flasks were subcultured or used for experiments (passages 28-33).

Prior to cell seeding, the microfluidic chips were rinsed with PBS and coated with fibronectin $\left(20 \mu \mathrm{g} \mathrm{m} \mathrm{L}^{-1}\right)$ for 3 hours at $37^{\circ} \mathrm{C}$. After flushing any air bubbles out, the chips were filled with EGM-2 and incubated for 2 hours. hCMEC/D3 cells were obtained from a confluent flask using $0.05 \%$ trypsin-EDTA (Gibco), suspended in fresh EGM-2 at $5 \cdot 10^{6}$ cells mL $^{-1}$ (corresponding to $2 \cdot 10^{5}$ cells $\mathrm{cm}^{-}$ ${ }^{2}$ ) and pipetted into the top channel of a microfluidic chip. After 1 hour of static incubation non-attached cells were washed away with EGM-2 and medium-filled pipette tips were inserted as reservoirs in all inlets. Medium was refreshed twice daily by inserting new medium-filled pipette tips in the inlets, replacing the medium inside the chip by gravity-driven flow.

At the end of an experiment the channels were washed with PBS and the cells were fixed with 3.7\% paraformaldehyde (Sigma Aldrich) in PBS for 30 minutes at room temperature (RT). The fixative was washed away with PBS and the cells were shortly incubated in permeabilization buffer (PB), which consisted of $0.1 \%(\mathrm{v} / \mathrm{v})$ Triton X-100 (Sigma Aldrich) and 1\% (m/m) bovine serum albumin (Sigma) in PBS. Then the cells were incubated for 2 hours at RT with mouse anti-human ZO-1 IgG (5 $\mu \mathrm{g} \mathrm{mL} \mathrm{m}^{-1}$ in PB; BD 
Transduction Laboratories). After washing three times with PBS, the cells were incubated for 1 hour at RT with goat anti-mouse IgG Alexa Fluor 488 (5 $\mu \mathrm{g} \mathrm{mL}^{-1}$ in PB; Invitrogen). After flushing three times with PBS, the nuclei were stained with NucBlue (1 drop per 500 LL PB; Ready Probes reagent, Molecular Probes, Life Technologies) for 30 min at RT, after which the channels were flushed 3 times with PBS. Chips were opened by removing the top part and electrodes, while keeping the membrane fixed to the bottom part. The bottom part was placed upside-down in PBS on a glass cover slip. The cells were imaged with phase contrast and fluorescence microscopy using the EVOS FL Cell Imaging System (Life Technologies; green filters (ex 470/22, em 510/42) for ZO-1 and blue filters (ex 357/44, em 447/60) for NucBlue).

\subsection{TEER measurements}

The TEER measurement setup consisted of a lock-in amplifier with a probe cable circuit (schematically shown in Suppl. Fig. 2), operated by a LabVIEW programme (designed, manufactured and programmed in-house). Proper functioning of this lock-in setup was verified with two commercial machines (SP-300 potentiostat, Bio-Logic Science Instruments; and Impedance/Gain-Phase analyser, type 4194A, Hewlett Packard; data not shown). Impedance spectra were recorded using an AC signal with 0.15 or 0.3 $V_{\text {RMS }}$, scanning from $200 \mathrm{~Hz}$ to $1 \mathrm{MHz}$. Each of the 100 logarithmically spaced measurement points was the average of 50 periods. Typical impedance spectra for a blank chip and a chip with cells are shown in Suppl. Fig. 1. From the resulting impedance spectra the resistances between the electrodes were determined from the resistive plateau at $10 \mathrm{kHz}$. Measurement errors due to changes in temperature and medium conductivity are minimized by refreshing the medium inside the chip and allowing it to reach room temperature prior to each measurement, according to established TEER protocols commonly used in literature.

The resistance between two electrodes is determined for all six combinations, denoted as $R_{i \rightarrow j}$ with $i$ and $j$ referring to the electrodes as numbered in Fig. 1. Since each measured resistance is the sum of the resistors in its path, as is shown in the equivalent resistive circuit presented in Fig. 1E, a system of six equations and five unknowns results. From this system of equations the values of all five resistors can be determined using Gaussian elimination, including the resistance of the cellular barrier and membrane $\left(R_{m}\right)$. The two solutions per unknown that resulted from these six equations with five unknowns were summed to obtain one solution that includes as many measurements as possible. The TEER $\left(\Omega \mathrm{cm}^{2}\right)$ is determined by normalizing $R_{m}$ to the culture area $A_{\text {cult }}\left(\mathrm{cm}^{2}\right)$ and by subtracting the TEER of the system prior to cell seeding $(\mathrm{t}=0)$. Thus Eq. 2 was used to calculate the TEER:

$$
\text { TEER }=A_{\text {cult }} \cdot R_{m}=A_{\text {cult }} \cdot \frac{1}{4}\left(R_{1 \rightarrow 2}+R_{1 \rightarrow 4}+R_{2 \rightarrow 3}+R_{3 \rightarrow 4}-2 R_{1 \rightarrow 3}-2 R_{2 \rightarrow 4}\right)\left[\Omega \mathrm{cm}^{2}\right] \quad \text { Eq. } 2
$$

The typical values for these resistances can be estimated using Eq. 1 presented in the introduction. A channel of cross-sectional area $A_{c h}=500 \mu \mathrm{m} \cdot 375 \mu \mathrm{m}=1.9 \cdot 10^{-7} \mathrm{~m}^{2}$ with two electrodes spaced by $l_{c h}=7 \mathrm{~mm}$, filled with culture medium with a specific conductivity of approximately $\sigma=1.6 \mathrm{~S} \mathrm{~m}^{-1}$ and thus a resistivity of $\rho=\frac{1}{1.6} \Omega \mathrm{m}=0.625 \Omega \mathrm{m}$, has an electrical resistance of $R_{c h}=$ $\rho \frac{l_{c h}}{A_{c h}}=0.625 \Omega m \cdot \frac{7 \cdot 10^{-3} \mathrm{~m}}{1.9 \cdot 10^{-7} \mathrm{~m}^{2}} \approx 2.3 \cdot 10^{4} \Omega$. In the same device, the resistance of a cell barrier with a TEER of $30 \Omega \mathrm{cm}^{2}(\mathrm{a}$ typical value for the hCMEC/D3 cell line (Weksler et al. 2013)), on a culture area of $A_{\text {cult }}=500 \cdot 500 \mu \mathrm{m}^{2}=2.5 \cdot 10^{-3} \mathrm{~cm}^{2}$ will have a resistance of $R_{\text {barrier }}=\frac{T E E R}{A_{\text {cult }}}=\frac{30 \Omega \mathrm{cm}^{2}}{2.5 \cdot 10^{-3} \mathrm{~cm}^{2}} \approx 1.2 \cdot 10^{4} \Omega$. This shows that the expected channel resistance is of the same order of magnitude or even larger than the expected cellular barrier resistance, which corresponds to our experimental results shown in Suppl. Fig. 1. This effect is even more pronounced when longer channels with smaller cross-sectional areas are used. Note that the effect of (especially long and shallow) channel geometries on the current distribution along the cellular barrier should be taken into account and, if needed, mathematically corrected when measuring TEER, as is discussed in the paper of Odijk et al. 
(2015). In our device the length and height of the culture area are comparatively small (500 $\mu \mathrm{m}$ and $375 \mu \mathrm{m}$, respectively) at the interface of the two channels, resulting in a local channel resistance of $R_{c h}=\rho \frac{l_{c h}}{A_{c h}}=0.625 \Omega \mathrm{m} \cdot \frac{500 \cdot 10^{-6} \mathrm{~m}}{1.9 \cdot 10^{-7} \mathrm{~m}^{2}} \approx 1.6 \cdot 10^{3} \Omega$ and a barrier resistance of $R_{\text {barrier }}=1.2 \cdot 10^{4} \Omega$, as was estimated before. Because of the relatively large difference between these resistances, we can assume that the current distribution is uniform across the cellular barrier. Therefore, it was not required to mathematically correct the TEER with respect to the current distribution in our device.

\section{Results and discussion}

Microfluidic chips contain small volumes and, as a result, they have high internal electrical resistances in comparison to the resistance of interest, the TEER. This means that very small variations in e.g. temperature and medium solute concentrations that affect the inherent resistance of the system (see Suppl. Fig. 3-4) can change the apparent TEER. To explore this effect, we performed an experiment in which we filled devices with EGM-2 cell culture medium and monitored the resistance during 15 days $(n=3)$. We subtracted the first measurement from all subsequent measurements, expecting to find a horizontal line at $0 \mathrm{k} \Omega$ for these blank chips. However, in the representative data of Fig. 2A it can be seen that there is a big spread in the measured impedances due to the large variations in resistance inherent to microfluidic systems, even though we used established TEER protocols to minimize the effect of changes in temperature and medium conductivity. When we used these six measurements between the electrode pairs to directly determine the membrane resistance $R_{m}$, as is shown in Fig. $2 \mathrm{~B}$, the variation is greatly reduced. The same is seen for all three chips, of which all data is shown in Suppl. Fig. 5A and 5B. Therefore, we concluded that our fourelectrode method enables the direct isolation of the membrane resistance, regardless of variations in the system. Next, we performed an experiment in which we cultured hCMEC/D3 cerebral endothelial cells inside chips and monitored barrier formation with TEER measurements during three days $(n=4)$. We subtracted a blank measurement (before adding the cells) from all subsequent measurements, expecting to find the increase resulting from biological changes in the barrier tightness. However, as can be seen in the representative data of Fig. 2C, different resistances resulted per electrode pair. With our 4-electrode method we were able to directly determine the resistance of the membrane plus cell barrier from these six measurements, as is shown in Fig. 2D. The same is seen for all four chips, of which all data is shown in Suppl. Fig. 5C and 5D. In conclusion, our newly developed method makes sure that the resistance of the cellular barrier and membrane are isolated from the system, diminishing the influence of variance of non-biological origin. 


\section{Blank chip}
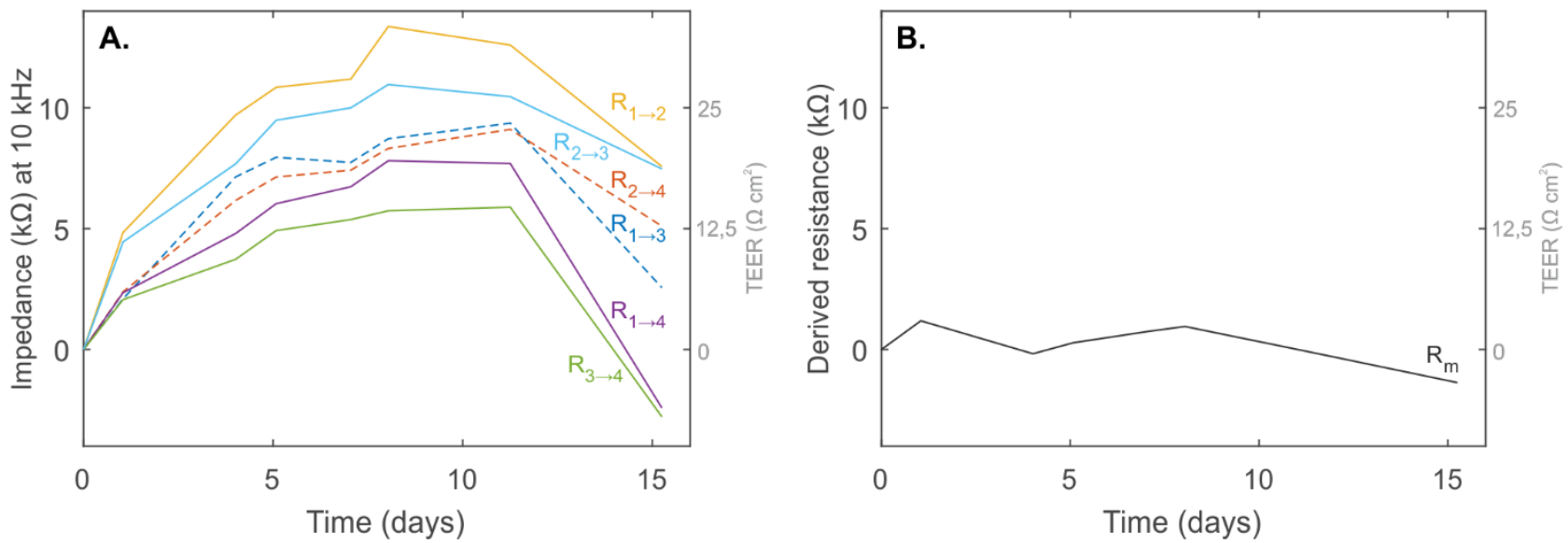

\section{Chip with hCMEC/D3 cells}
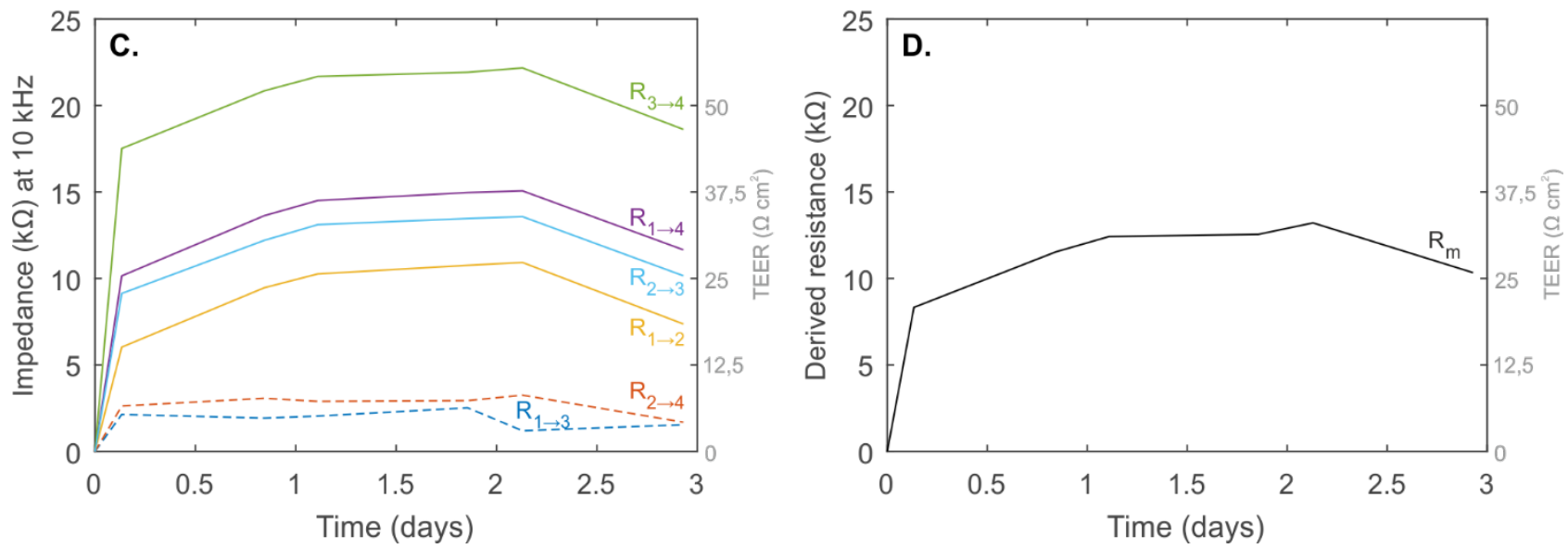

Fig. 2 A. Measurements of the membrane resistance in a blank chip (filled with EGM-2 culture medium). Measured impedance at $10 \mathrm{kHz}$ for each electrode pair. Dashed lines correspond to measurements only through the microfluidic channels, solid lines indicate that the membrane was included in the measured path. The first measurement (at $O$ days) was subtracted from all subsequent measurements. $A$ horizontal line at $0 \mathrm{k} \Omega$ was expected, but large variation is observed. For easy comparison the resistance is also displayed in units of TEER $\left(\Omega \mathrm{cm}^{2}\right)$. B. The calculated membrane resistance $R_{m}$ has much less variation than the measured resistances. The first measurement was subtracted from all subsequent measurements.

C. TEER measurements in a chip with hCMEC/D3 cells. Measured impedance at $10 \mathrm{kHz}$ for each electrode pair. The blank measurement (at 0 days, before adding cells) was subtracted from all subsequent measurements. There is large variation between the electrode pairs. For easy comparison the resistance is also displayed in units of $\operatorname{TEER}\left(\Omega \mathrm{cm}^{2}\right)$. D. By calculating $R_{m}$ the resistance of the membrane and cells is isolated. The first measurement was subtracted from all subsequent measurements. 


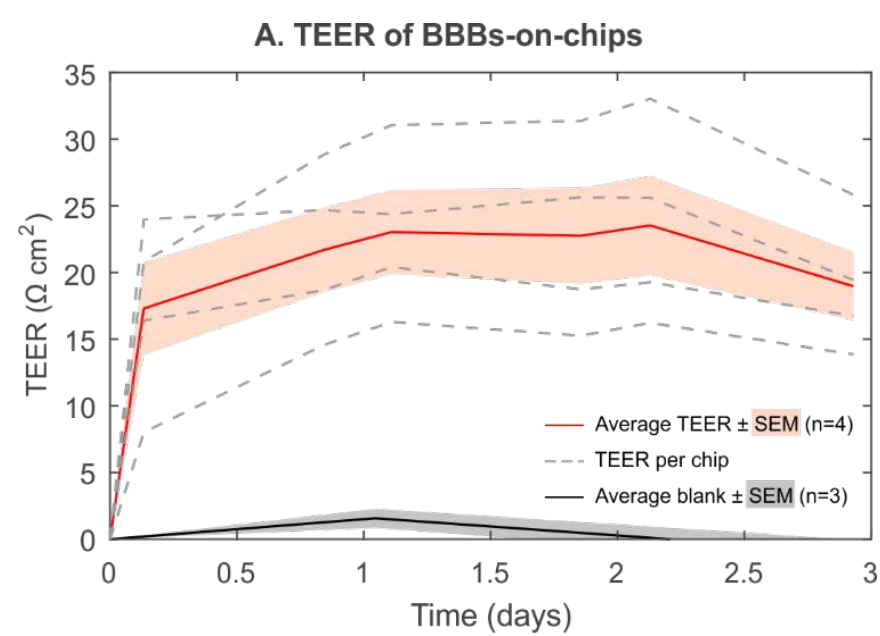

B. Fluorescence microscopy of BBB-on-chip

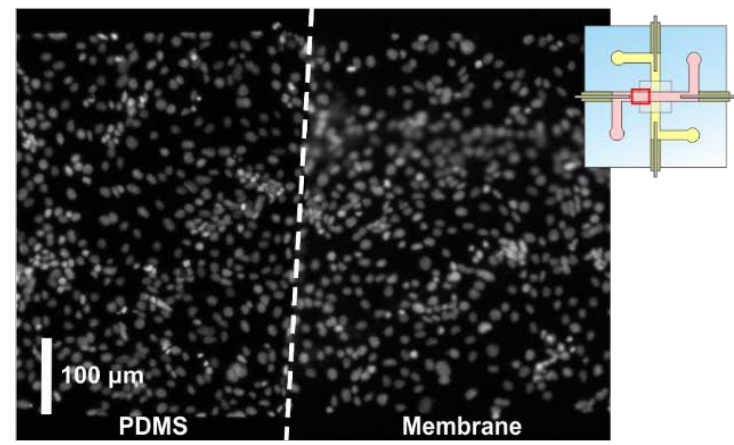

C. Fluorescence microscopy of tight junctions

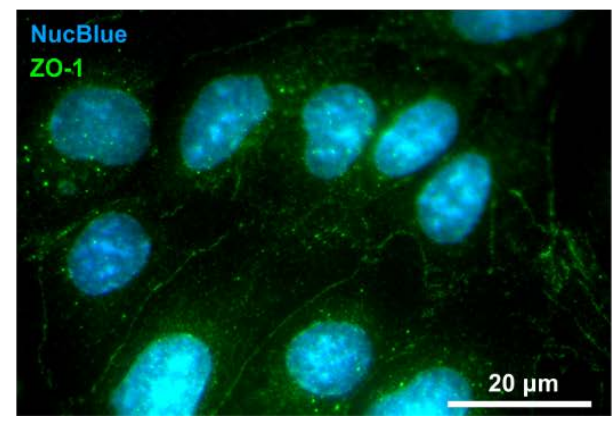

Fig. 3 A. Measured TEER values of four BBBs-on-chips during three days compared to blank chips. SEM = standard error of the mean. $\boldsymbol{B}$. Fluorescence microscopy of NucBlue stained nuclei confirmed the presence of a continuous monolayer of endothelial cells inside the chips. The inset shows where the microscopic image was taken. $\mathbf{C}$. Fluorescence microscopy confirmed the presence of tight junction protein ZO-1.

in channel resistance due to non-biological factors, e.g. changes in temperature, medium conductance, electrode position and the presence of air bubbles. This makes it difficult to draw quantitative conclusions and compare results.

In this paper we showed that the TEER in organs-on-chips can be directly determined with four electrodes and six measurements independent of changes in non-biological factors in the system. With less than four electrodes the TEER cannot be determined directly, because there are more unknowns than measurement possibilities. This method is suitable for any chip with two channels in each of which two electrodes can be inserted, while still being compatible with the simple measurement setup that is used for conventional 2-electrode TEER measurements. In conclusion, our newly developed method to determine TEER with four
Next, the TEER was monitored of four BBBs-on-chips, as shown in Fig. 3A. The average TEER of $22 \Omega \mathrm{cm}^{2}$ in the plateau from day 13 and the biological variation (standard error of the mean $(\mathrm{SEM})=$ $1.3 \Omega \mathrm{cm}^{2}$ ) was close to other TEER values reported for hCMEC/D3 cells (Weksler et al. 2013). Microscopic inspection of the cells after terminating the experiment showed that there was a continuous cell layer (representative image shown in Fig. 3B and Suppl. Fig. 6) and the cells expressed tight junction proteins (Fig. 3C), contributing to this increased TEER. For barrier-forming tissues, one may expect higher TEER values and more ZO-1 expression. While the hCMEC/D3 cell line is a comparatively reproducible source of human endothelial cells that is often used in literature, it is known to poorly develop tight junctions (Abbott et al. 2014). In our study, however, we preferred biological relevance over the barrier formation capabilities and chose to work with this human cell line. As a next step we want to expose the hCMEC/D3 cells to increase barrier formation (Weksler et al. 2013).

\section{Conclusion}

TEER is a non-invasive indicator of the tightness of a cellular barrier that is widely used in conventional Transwell systems as well as in organs-on-chips. Electrodes are conveniently inserted in the chip's outlets because integrated electrodes close to the cellular barrier in organs-on-chips require specialized cleanroom processes and, more importantly, often hamper visual inspection of the cells inside the device. Unfortunately, conventional TEER measurements are often prone to variation and imprecision due the high resistance of the fluid-filled microfluidic channels.

Therefore, the apparent TEER is greatly influenced by any change cells to shear stress and co-culture them with BBB-associated 
electrodes without the need for separate measurements of empty chips is expected to be easily incorporated in existing organ-onchip systems and would provide more useful and meaningful TEER measurements in organ-on-chip applications.

\section{Acknowledgements}

We gratefully acknowledge Johan Bomer for performing all cleanroom processes and Mathijs Bronkhorst for fruitful discussions and assistance with data representation.

This research was funded by the SRO Biomedical Microdevices and the SRO Organs-on-chips, MIRA Institute for Biomedical Technology and Technical Medicine, University of Twente, the Netherlands, as well as partially by VESCEL, ERC Advanced Grant to A. van den Berg.

\section{References}

Abbott, N.J., Dolman, D.M., Yusof, S., Reichel, A., 2014. In Vitro Models of CNS Barriers. In: Hammarlund-Udenaes, M., de Lange, E.C.M., Thorne, R.G. (Eds.), Drug Delivery to the Brain, pp. 163-197. Springer New York.

Abbott, N.J., Patabendige, A.A., Dolman, D.E., Yusof, S.R., Begley, D.J., 2010. Structure and function of the blood-brain barrier. Neurobiology of disease 37(1), 13-25.

Bhatia, S.N., Ingber, D.E., 2014. Microfluidic organs-on-chips. Nature biotechnology 32(8), 760-772.

Booth, R., Kim, H., 2012. Characterization of a microfluidic in vitro model of the blood-brain barrier (mu BBB). Lab Chip 12(10), 1784-1792.

Brown, J.A., Pensabene, V., Markov, D.A., Allwardt, V., Neely, M.D., Shi, M., Britt, C.M., Hoilett, O.S., Yang, Q., Brewer, B.M., Samson, P.C., McCawley, L.J., May, J.M., Webb, D.J., Li, D., Bowman, A.B., Reiserer, R.S., Wikswo, J.P., 2015. Recreating bloodbrain barrier physiology and structure on chip: A novel neurovascular microfluidic bioreactor. Biomicrofluidics 9(5), 054124.

Cardoso, F.L., Brites, D., Brito, M.A., 2010. Looking at the blood-brain barrier: molecular anatomy and possible investigation approaches. Brain research reviews 64(2), 328-363.

Chueh, B.-H., Huh, D., Kyrtsos, C.R., Houssin, T., Futai, N., Takayama, S., 2007. Leakage-free bonding of porous membranes into layered microfluidic array systems. Analytical chemistry 79(9), 3504-3508.

Deosarkar, S.P., Prabhakarpandian, B., Wang, B., Sheffield, J.B., Krynska, B., Kiani, M.F., 2015. A Novel Dynamic Neonatal BloodBrain Barrier on a Chip. PLoS ONE 10(11), e0142725.

Douville, N.J., Tung, Y.-C., Li, R., Wang, J.D., El-Sayed, M.E., Takayama, S., 2010. Fabrication of two-layered channel system with embedded electrodes to measure resistance across epithelial and endothelial barriers. Analytical chemistry 82(6), 2505-2511. Ferrell, N., Desai, R.R., Fleischman, A.J., Roy, S., Humes, H.D., Fissell, W.H., 2010. A microfluidic bioreactor with integrated transepithelial electrical resistance (TEER) measurement electrodes for evaluation of renal epithelial cells. Biotechnology and bioengineering 107(4), 707-716.

Griep, L.M., Wolbers, F., de Wagenaar, B., ter Braak, P.M., Weksler, B.B., Romero, I.A., Couraud, P.O., Vermes, I., van der Meer, A.D., van den Berg, A., 2013. BBB ON CHIP: microfluidic platform to mechanically and biochemically modulate blood-brain barrier function. Biomedical Microdevices 15(1), 145-150.

Huh, D., Matthews, B.D., Mammoto, A., Montoya-Zavala, M., Hsin, H.Y., Ingber, D.E., 2010. Reconstituting organ-level lung functions on a chip. Science 328(5986), 1662-1668. 
Kim, H.J., Huh, D., Hamilton, G., Ingber, D.E., 2012. Human gut-on-a-chip inhabited by microbial flora that experiences intestinal peristalsis-like motions and flow. Lab Chip 12(12), 2165-2174.

Moraes, C., Mehta, G., Lesher-Perez, S., Takayama, S., 2012. Organs-on-a-Chip: A Focus on Compartmentalized Microdevices. Annals of Biomedical Engineering 40(6), 1211-1227.

Odijk, M., van der Meer, A.D., Levner, D., Kim, H.J., van der Helm, M.W., Segerink, L.I., Frimat, J.-P., Hamilton, G.A., Ingber, D.E., van den Berg, A., 2015. Measuring direct current trans-epithelial electrical resistance in organ-on-a-chip microsystems. Lab Chip 15(3), 745-752.

Srinivasan, B., Kolli, A.R., Esch, M.B., Abaci, H.E., Shuler, M.L., Hickman, J.J., 2015. TEER Measurement Techniques for In Vitro Barrier Model Systems. Jala 20(2), 107-126.

Thuenauer, R., Rodriguez-Boulan, E., Romer, W., 2014. Microfluidic approaches for epithelial cell layer culture and characterisation. Analyst 139(13), 3206-3218.

van der Helm, M.W., van der Meer, A.D., Eijkel, J.C.T., van den Berg, A., Segerink, L.I., 2016. Microfluidic organ-on-chip technology for blood-brain barrier research. Tissue Barriers(just-accepted), 00-00.

van der Meer, A.D., van den Berg, A., 2012. Organs-on-chips: breaking the in vitro impasse. Integrative Biology 4(5), 461-470. van der Meer, A.D., Wolbers, F., Vermes, I., van den Berg, A., 2014. Blood-brain Barrier (BBB): An Overview of the Research of the Blood-brain Barrier Using Microfluidic Devices. In: van den Berg, A., Segerink, L.I. (Eds.), Microfluidics for Medical Applications, pp. 40-56.

Walter, F.R., Valkai, S., Kincses, A., Petneházi, A., Czeller, T., Veszelka, S., Ormos, P., Deli, M.A., Dér, A., 2016. A versatile labon-a-chip tool for modeling biological barriers. Sensors and Actuators B: Chemical 222, 1209-1219.

Weksler, B., Romero, I.A., Couraud, P.-O., 2013. The hCMEC/D3 cell line as a model of the human blood brain barrier. Fluids Barriers CNS 10(1), 16.

Weksler, B., Subileau, E., Perriere, N., Charneau, P., Holloway, K., Leveque, M., Tricoire-Leignel, H., Nicotra, A., Bourdoulous, S., Turowski, P., 2005. Blood-brain barrier-specific properties of a human adult brain endothelial cell line. The FASEB journal 19(13), 1872-1874. 

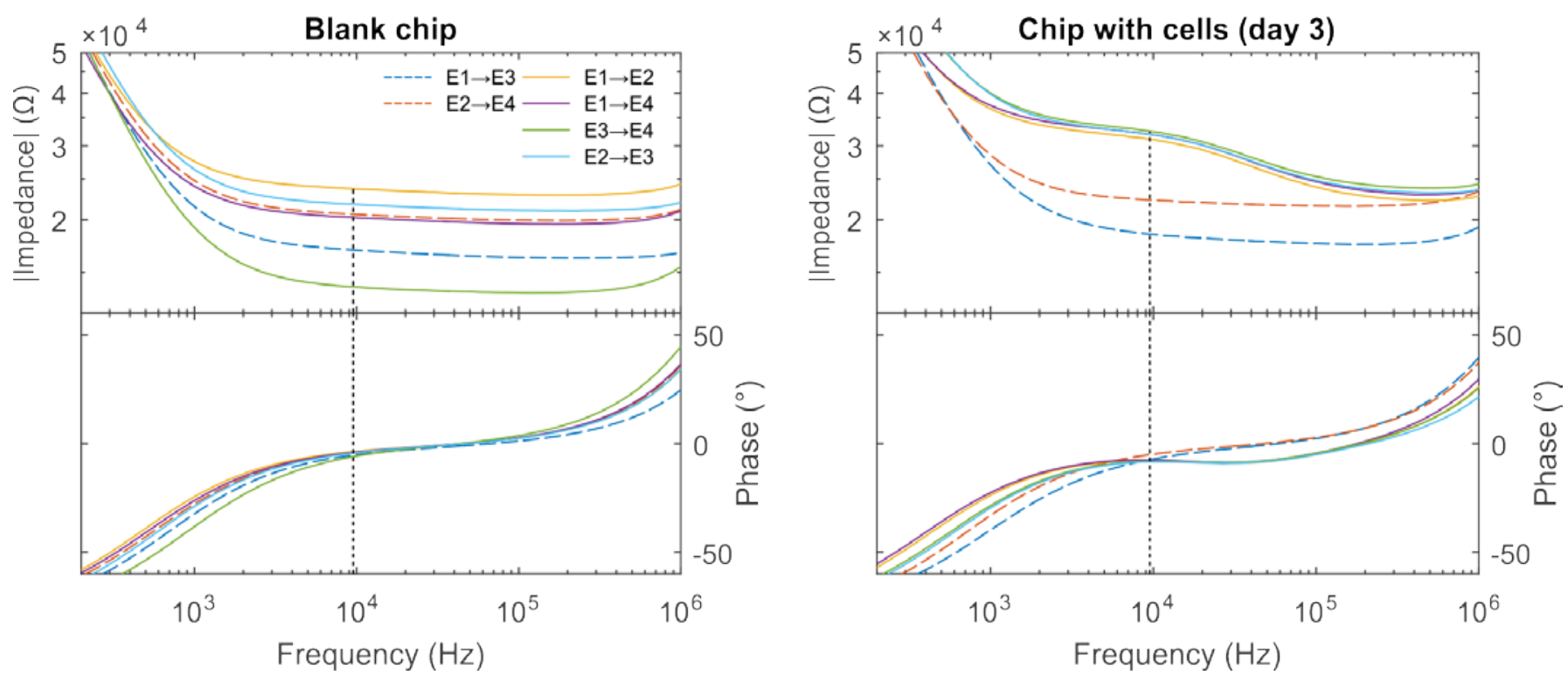

Suppl. Fig. 1 Typical impedance spectra of the BBB chip. The impedance magnitude $(\Omega)$ and phase $\left({ }^{\circ}\right)$ are plotted against the measurement frequency $(\mathrm{Hz})$ for a blank chip (only EGM-2 culture medium, before adding cells) and for a chip in which hCMEC/D3 cells were cultured for 3 days. A clear difference between the two sets of spectra is the extra resistive plateau seen in the chip with cells. This is caused by the resistance and capacitance of the cellular barrier. As readout frequency $10 \mathrm{kHz}$ was chosen, since it lies in this extra resistive plateau of the cells, while it is not being influenced by the double layer capacitance of the electrodes (regime up to $3 \mathrm{kHz}$ ). Note that the magnitude of the impedance at $10 \mathrm{kHz}$ corresponds well to the calculated values of the channel resistance $R_{c h}=23 \mathrm{k} \Omega(E 1 \rightarrow E 3$ and $E 2 \rightarrow E 4$ in right figure) and the summed channel and barrier resistance $R_{c h}+R_{\text {barrier }}=23 k \Omega+12 k \Omega=35 k \Omega(E 1 \rightarrow E 2, E 1 \rightarrow E 4, E 3 \rightarrow E 4$ and $E 2 \rightarrow E 3$ in right figure $)$.
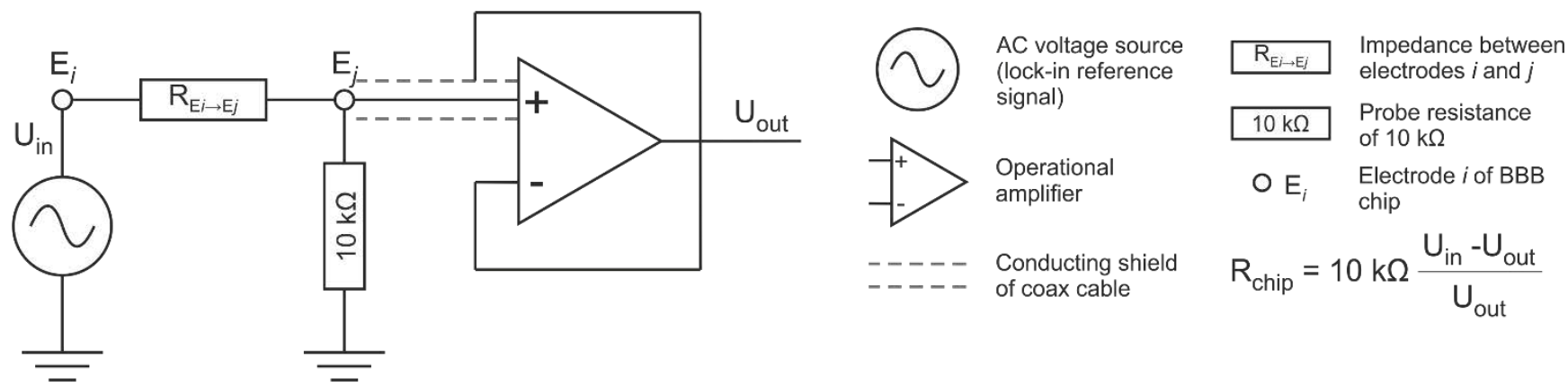

Suppl. Fig. 2 Probe cable used in combination with lock-in amplifier in the TEER measurement setup. The probe resistance was $10 \mathrm{k} \Omega$, optimized for use in our BBB-on-chip. The impedance between electrode $\mathrm{i}$ and $\mathrm{j}$ can be calculated using the probe resistance, the applied voltage $\mathrm{U}_{\text {in }}$ and the measured voltage response $\mathrm{U}_{\text {out }}$. 
A. Influence of temperature

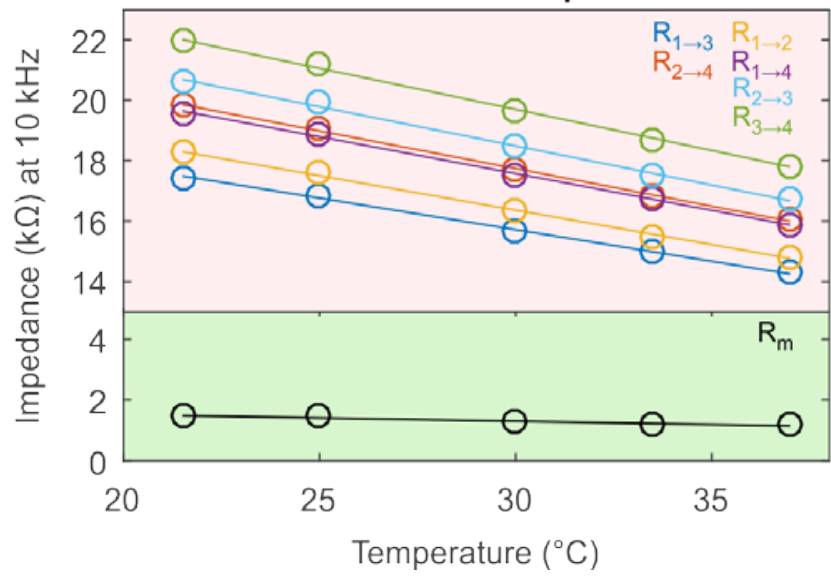

B. Influence of ion concentration

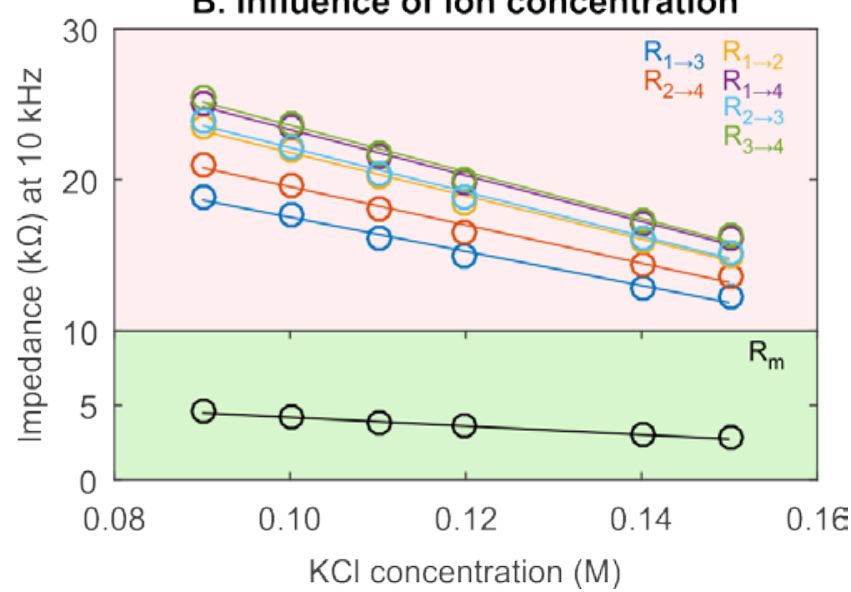

Suppl. Fig. 3 A. Influence of temperature on the measured impedance in a chip. A chip was filled with EGM-2 cell culture medium and taped to a microscope heating stage (Leica MA TS). The impedance spectra were measured for each electrode pair at different temperatures after allowing the system 10-15 min to equilibrate to that temperature. The resulting impedances at $10 \mathrm{kHz}$ are plotted here (colored lines), showing a clear decrease in resistance as a result of increasing temperature. The calculated $R_{m}$ (black) shows an 11-fold decrease in sensitivity for temperature, from $-244 \Omega{ }^{\circ} \mathrm{C}^{-1}$ to $-21 \Omega{ }^{\circ} \mathrm{C}^{-1}$ (linearly approximated). B. Influence of ion concentration on the measured impedance in a chip. $A$ chip was filled with $\mathrm{KCl}$ solutions with concentrations around $0.11 \mathrm{M}$, which has the same conductivity as EGM-2 culture medium (1.3 S m at 20 ${ }^{\circ} \mathrm{C}$, as measured with the Mettler Toledo SevenMulti Conductivity meter). For each measurement, the chip was flushed thoroughly and taped to a microscope heating stage set at $25^{\circ} \mathrm{C}$ (Leica MA TS). After allowing the system 5-10 min to equilibrate the impedance spectra were measured for each electrode pair at the increasing ion concentrations. The resulting impedances at $10 \mathrm{kHz}$ are plotted here (colored lines), showing a clear decrease in resistance as a result of increasing ion concentration. The calculated $R_{m}$ (black) shows a 5-fold decrease in sensitivity for ion concentration, from $-139 \Omega \mathrm{mM}^{-1}$ to $-29 \Omega \mathrm{mM}^{-1}$ (linearly approximated).

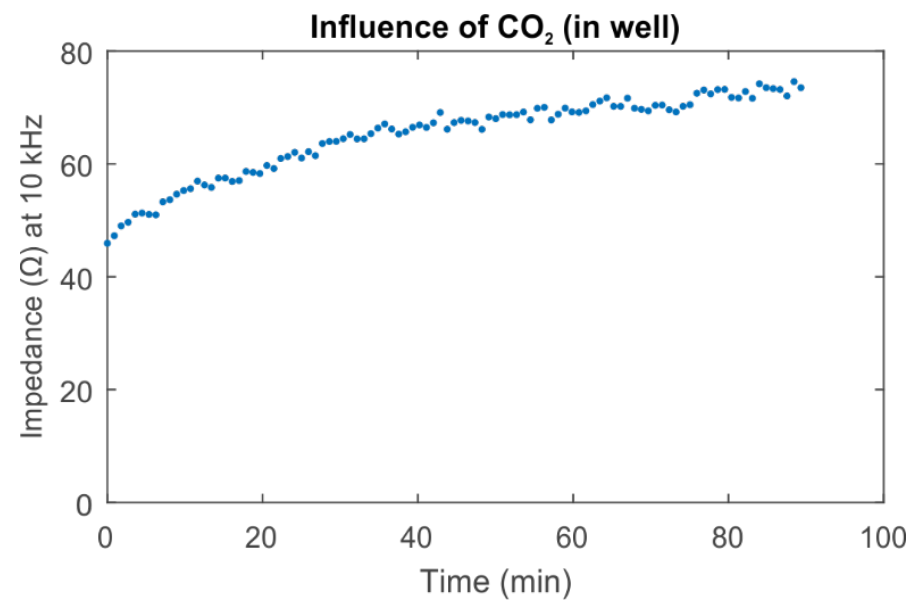

Suppl. Fig. 4 Influence of $\mathrm{CO}_{2}$ concentration on the measured impedance in a well. A well in a 12-wells plate was filled with $2 \mathrm{~mL} E G M-2$ culture medium and left for 22 hours in an incubator $\left(37^{\circ} \mathrm{C}, 5 \% \mathrm{CO}_{2}\right)$ to equilibrate. Then the plate was exposed to normal atmosphere and impedance spectra were recorded during 90 minutes on a microscope heating stage set to $37^{\circ} \mathrm{C}$ (Leica MA TS). There was a $\pm 60 \%$ increase in impedance at $10 \mathrm{kHz}$ between $0 \mathrm{~min}$ and $90 \mathrm{~min}$, indicating that there was a decrease in amount of ions resulting from the lower $\mathrm{CO}_{2}$ concentration. 


\section{Blank chips}
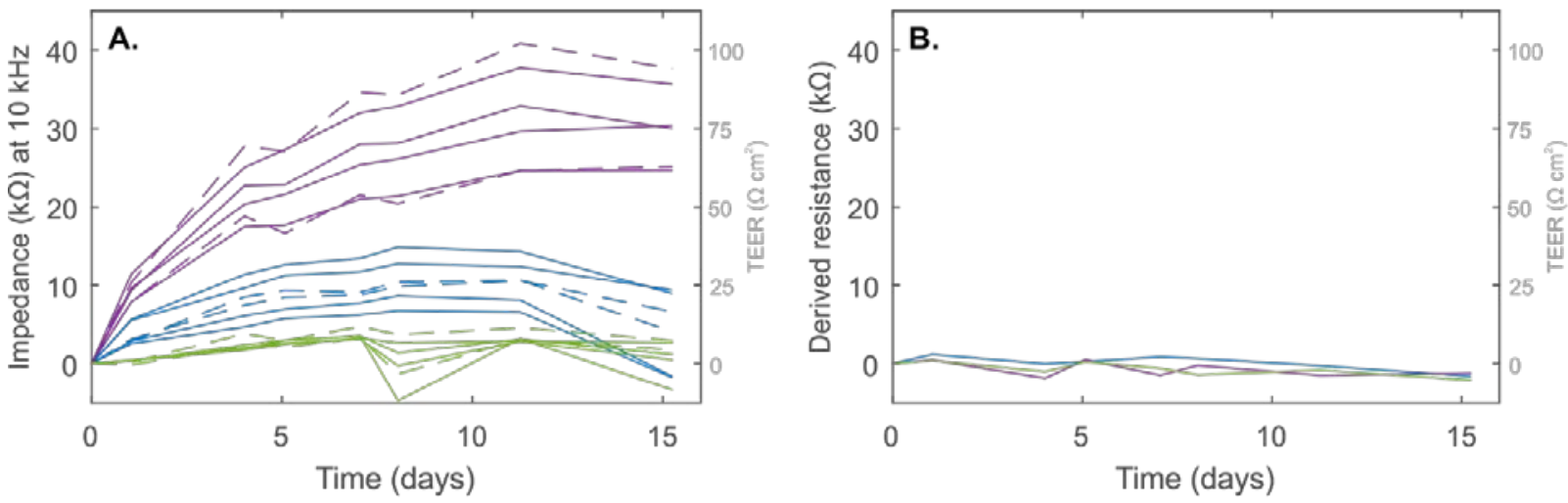

Chips with hCMEC/D3 cells
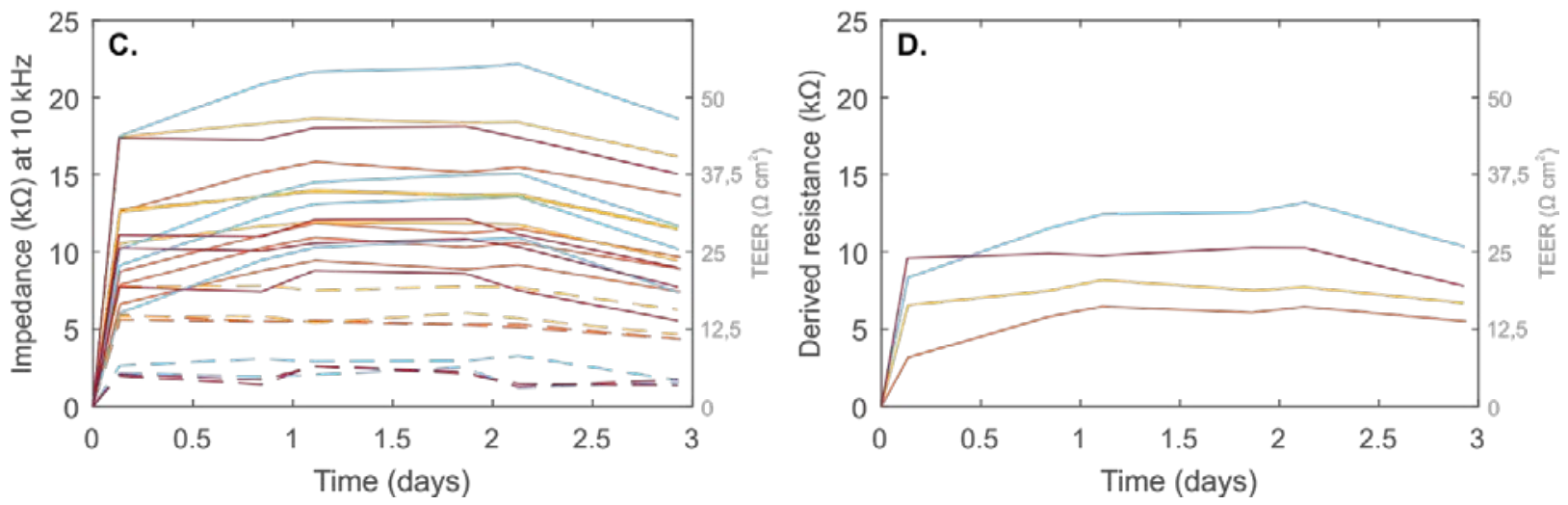

Suppl. Fig. 5 A. Measurements of the membrane resistance in three blank chips (filled with EGM-2 culture medium). Measured impedances at $10 \mathrm{kHz}$ for each electrode pair. Dashed lines correspond to measurements only through the microfluidic channels, solid lines indicate that the membrane was included in the measured path. The first measurement (at 0 days) was subtracted from all subsequent measurements. A horizontal line at $0 \mathrm{k} \Omega$ was expected, but large variation is observed in all three chips. For easy comparison the resistance is also displayed in units of TEER $\left(\Omega \mathrm{cm}^{2}\right)$. B. The calculated membrane resistance $R_{m}$ has much less variation than the measured resistances. The first measurement was subtracted from all subsequent measurements.

C. TEER measurements in four chips with hCMEC/D3 cells. Measured impedances at $10 \mathrm{kHz}$ for each electrode pair. The blank measurement (at 0 days, before adding cells) was subtracted from all subsequent measurements. There is large variation between the electrode pairs. For easy comparison the resistance is also displayed in units of TEER $\left(\Omega \mathrm{cm}^{2}\right)$. D. By calculating $R_{m}$ the resistance of the membrane and cells of each chip is isolated. The first measurement was subtracted from all subsequent measurements.

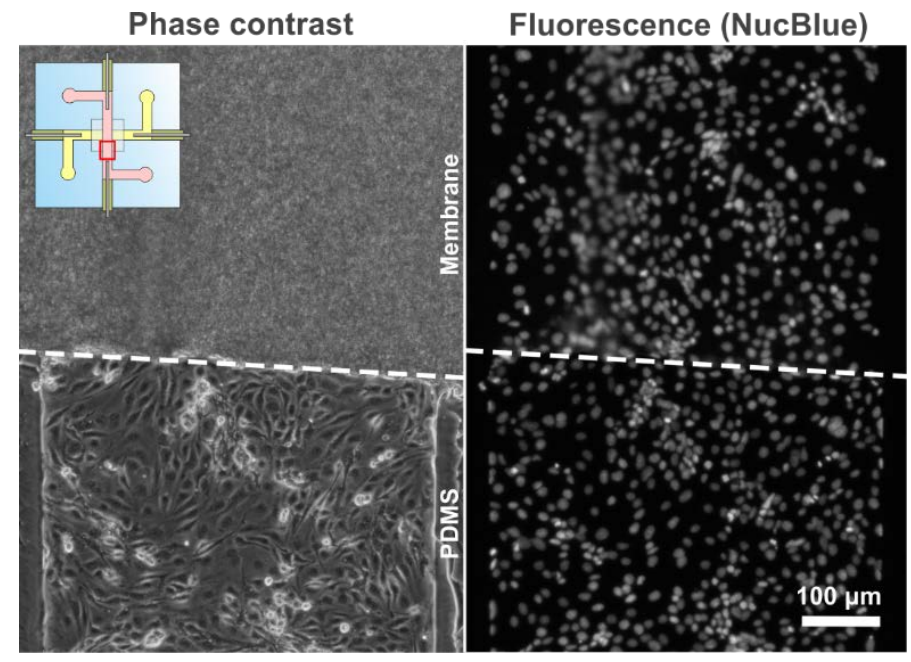

Suppl. Fig. 6 Phase contrast and fluorescence images of endothelial cell layer in the chip. Due to the high contrast of the polycarbonate membrane the cells growing on the membrane cannot be viewed using phase contrast, but fluorescently stained nuclei confirmed the presence of a continuous monolayer of endothelial cells. The inset shows where the images were taken in the chip. 